\title{
The local recognition of reflection graphs of spherical Coxeter groups
}

\author{
Ralf Gramlich · Jonathan I. Hall • Armin Straub
}

Received: 14 August 2008 / Accepted: 19 August 2009 / Published online: 2 September 2009

(C) Springer Science+Business Media, LLC 2009

\begin{abstract}
Based on the third author's thesis (arXiv:0805.2403) in this article we complete the local recognition of commuting reflection graphs of spherical Coxeter groups arising from irreducible crystallographic root systems.
\end{abstract}

Keywords Local recognition of graphs · Coxeter groups

\section{Introduction}

Given a connected graph one may ask to which extent it is determined by its local graphs, that is, by the induced subgraphs on the vertices adjacent to a particular vertex. This local recognition of graphs has been studied extensively in the literature, for

The first author gratefully acknowledges a Heisenberg Fellowship by the Deutsche Forschungsgemeinschaft.

The second author gratefully acknowledges partial support provided by the National Science Foundation (USA).

R. Gramlich $(\bowtie)$

FB Mathematik, Technische Universität Darmstadt, Schloßgartenstraße 7, Darmstadt 64289, Germany

e-mail: gramlich@mathematik.tu-darmstadt.de

R. Gramlich

School of Mathematics, University of Birmingham, Edgbaston, Birmingham, B15 2TT, UK

e-mail: ralfg@maths.bham.ac.uk

J.I. Hall

Department of Mathematics, Michigan State University, East Lansing, Michigan 48824, USA

e-mail: jhall@math.msu.edu

A. Straub

Department of Mathematics, Tulane University, 6823 St. Charles Ave, New Orleans, LA 70118, USA

e-mail: math@arminstraub.com 
instance in $[4,9,18,22,30,31]$ to mention a few; see also $[3,6,15]$. A particularly guiding example for the topic of the present article is the local recognition of the Kneser graphs studied in [14] and [16].

We are interested in the local recognition of Weyl graphs, i.e., graphs on the reflections of Coxeter groups with the commutation relation as adjacency. A combination of our findings with results from $[4,16,18]$ yields the following recognition result.

Main Theorem The following are true up to isomorphism.

- A Weyl graph of type $A_{n}(n \geqslant 8)$, type $B_{n}, C_{n}(n=3$ or $n \geqslant 5)$, type $D_{n}(n \geqslant 9)$, or type $E_{7}$ is uniquely determined, as a connected graph, by its local graphs.

- A Weyl graph of type $A_{6}, A_{7}, D_{7}, D_{8}, E_{6}, E_{8}$ is uniquely determined by its local graphs and its size.

- The Weyl graph $W$ of type $F_{4}$ and its twisted copy (defined at the end of section 4.1) are the only bichromatic graphs of size 24 with local graphs like W.

The remaining small Weyl graphs of type $A_{n}$ as well as those of types $I_{2}(m), G_{2}$, $H_{3}, H_{4}$ are locally a disjoint union of complete graphs. The graphs of type $D_{n}$ are obtained as doubles of those of type $A_{n-1}$, so that local recognition results for type $A_{n}$ transfer to $D_{n}$. Finally, types $B_{4}$ and $C_{4}$ are treated in Remark 12.

The local recognition of the Weyl graphs of type $A_{7}, E_{6}$ and $E_{8}$ has been established in the fundamental work [4]. The case of $A_{6}$, for which the Weyl graph is locally the Petersen graph, has been studied in [14]. Weyl graphs of types $A_{n}$ and $E_{n}$ which are locally cotriangular have been treated in [18]. The local recognition of types $B_{n}$ and $C_{n}$ is proved in Theorem 5 . The Weyl graph of type $F_{4}$ is not uniquely determined by its local graphs (Corollary 11). We nevertheless characterize this Weyl graph as one of two tightest graphs with the prescribed local structure (Theorem 16). In the last section we turn to group theoretical applications of local recognition results for Weyl graphs.

\section{Local recognition of graphs}

All graphs considered in this text are simple and undirected. We use $\perp$ to denote adjacency, and our notation for operations on graphs like the Cartesian product or joins follows [17]. Let $\Gamma$ be a graph, and $x \in \Gamma$ a vertex. We write $x^{\perp}$ to denote the set of neighbors of $x$, that is, the set of vertices adjacent to $x$. Likewise, for $X \subseteq \Gamma$ we write $X^{\perp}=\bigcap_{x \in X} x^{\perp}$. The induced subgraph on $x^{\perp}$ is called the local graph at $x$. A graph $\Gamma$ is said to be locally homogeneous, if there exists a graph $\Delta$ such that each local graph of $\Gamma$ is isomorphic to $\Delta$. In this case, $\Gamma$ is said to be locally $\Delta$, and $\Delta$ is referred to as the local graph of $\Gamma$. If $\Gamma$ is locally homogeneous, then we denote its local graph by $\Delta(\Gamma)$.

In this article we are interested in the problem of characterizing a connected locally homogeneous graph in terms of its local graph. We say that a connected locally homogeneous graph $\Gamma$ is locally recognizable, if up to isomorphism $\Gamma$ is the only connected graph that is locally $\Delta(\Gamma)$. In case $\Lambda$ is another locally homogeneous graph such that $\Delta(\Lambda) \cong \Delta(\Gamma)$ we say that $\Lambda$ is locally like $\Gamma$. 
The above terminology naturally extends to bichromatic graphs. For reasons that become clear later, we distinguish the vertices of a bichromatic graph as short versus long. All morphisms between bichromatic graphs are understood to preserve this distinction. We say that a bichromatic graph is locally homogeneous, if the local graphs at short vertices are all isomorphic to some bichromatic graph $\Delta_{s}$ and the local graphs at long vertices are all isomorphic to some bichromatic graph $\Delta_{\ell}$. In this case we say that $\Delta_{S}$ is the short local graph of $\Gamma$ and that $\Delta_{\ell}$ is the long local graph of $\Gamma$. If $\Gamma$ is a bichromatic locally homogeneous graph, then we denote its short local graph by $\Delta_{S}(\Gamma)$ and its long local graph by $\Delta_{\ell}(\Gamma)$. If $\Lambda$ is another bichromatic locally homogeneous graph such that the short as well as the long local graphs of $\Lambda$ and $\Gamma$ are isomorphic as bichromatic graphs, then we say that $\Lambda$ is locally like $\Gamma$. Finally, given a graph $\Gamma$ we denote with $\Gamma^{s}$ and $\Gamma^{\ell}$ the bichromatic graphs obtained from $\Gamma$ with all vertices treated as short respectively long.

One easily verifies that the Kneser graph $K(n, k)$ is locally homogeneous with local graph $K(n-k, k)$. The second author proved in [16] that for $n$ sufficiently large compared to $k$ the Kneser graphs are locally recognizable; for $k=2$, it sufficies to require $n \geq 7$. In [14] he classified the three connected graphs which are locally the Petersen graph $K(5,2)$. The classification of graphs that are locally $K(6,2)$ is contained in [4].

Theorem $1([4,14,16])$ Let $k \geqslant 1$, and $\Gamma$ be a connected graph that is locally $K(n, k)$.

- If $n \geqslant 3 k+1$ then $\Gamma \cong K(n+k, k)$.

- If $(n, k)=(5,2)$ then $\Gamma$ is isomorphic to one of the graphs $K(7,2), 3 \cdot K(7,2)$, or $\Sigma L_{2,25}$. In particular, $|\Gamma| \in\{21,63,65\}$.

- If $(n, k)=(6,2)$ then $\Gamma$ is isomorphic to one of the graphs $K(8,2), \mathcal{S} p_{6}(2)$ minus $\{x\} \cup x^{\perp}$ for some $x$, or $\mathcal{N}_{6}^{-}(2)$. In particular, $|\Gamma| \in\{28,32,36\}$.

Here, the graph $3 \cdot K(7,2)$ is the 3 -fold cover of $K(7,2)$, and $\Sigma L_{2,25}$ is the graph on the conjugates of the unique non-trivial field automorphism of $\mathbb{F}_{25}$ in the special semilinear group $\Sigma L(2,25)$ with two elements adjacent whenever they commute. More details can be found in [14]. Further, the graph $\mathcal{S} p_{2 n}(2)$ is the graph on the nonzero vectors of $V=\mathbb{F}_{2}^{2 n}$ with two vectors adjacent whenever they are perpendicular with respect to a non-degenerate symplectic form $B$ on $V$. Up to isomorphism there are only two quadratic forms $Q^{+}$and $Q^{-}$, corresponding to maximal or minimal Witt index, on $V$ that $B$ is associated to, and the graph $\mathcal{N}_{2 n}^{\varepsilon}(2)$ is the induced subgraph of $\mathcal{S} p_{2 n}(2)$ on the vectors that are non-singular under $Q^{\varepsilon}$. For more details about these graphs we refer to [18].

Ernest E. Shult and the second author actually proved a lot more in [18]. They characterize the graphs that are locally cotriangular in the following sense. A graph is said to be cotriangular, if every pair $x, y$ of non-adjacent vertices is contained in a cotriangle, that is, a 3 -coclique $\{x, y, z\}$ such that every other vertex is adjacent to either all or exactly one of the vertices $x, y, z$. Observe that a join $\Gamma+\Lambda$ is cotriangular if and only if both $\Gamma$ and $\Lambda$ are. Denote with $\Gamma^{*}$ the reduced graph of $\Gamma$, that is, the graph on the equivalence classes of vertices of $\Gamma$ with the same closed neighborhood and two classes adjacent whenever some representatives are adjacent. Then $\Gamma$ is 
cotriangular if and only if $\Gamma^{*}$ is. A graph $\Gamma$ is called completely reduced in this context whenever $\Gamma^{*}=\Gamma$ and $\Gamma$ can not be decomposed into $\Gamma_{1}+\Gamma_{2}$ with non-empty $\Gamma_{1}, \Gamma_{2}$. A classification of all cotriangular graphs is given by the following theorem due to Ernest E. Shult.

Theorem 2 ([27]) A finite completely reduced graph is cotriangular if and only if it is isomorphic to one of the graphs

$$
K(n, 2), \quad n \geqslant 2 ; \quad \mathcal{S}_{p_{2 n}}(2), \quad n \geqslant 2 ; \quad \mathcal{N}_{2 n}^{\varepsilon}(2), \quad \varepsilon= \pm 1, n \geqslant 3 .
$$

The graphs $K(2,2) \cong K_{1}$ and $K(3,2) \cong \overline{K_{3}}$ are considered degenerate. Let $\mathcal{D}$ denote the set of graphs $\Gamma$ such that $\Gamma^{*}$ is a finite completely reduced cotriangular graph. If $\mathcal{G}$ is a collection of graphs, then we say that a graph $\Gamma$ is locally $\mathcal{G}$ if for each $x \in \Gamma$ the local graph at $x$ is isomorphic to some graph of $\mathcal{G}$.

Theorem 3 ([18], Main Theorem) Let $\Gamma$ be connected and locally $\mathcal{D}$. Then either $\Gamma$ is locally $\left\{K_{1}, \overline{K_{3}}\right\}$ or $\Gamma$ is isomorphic to one of the following graphs

- $K(n, 2)$ where $n \geqslant 7$,

- $\mathcal{S} p_{2 n}(2)$ possibly with a polar subspace deleted,

- $\mathcal{H}_{2 n}^{\varepsilon}(T), \mathcal{G}_{2 n}^{\varepsilon}$,

- $3 \cdot K(7,2), \Sigma L_{2,25}$, or $\mathcal{N}_{6}^{+}(3)$.

The graphs $\mathcal{H}_{2 n}^{\varepsilon}(T), \mathcal{G}_{2 n}^{\varepsilon}$ are derived from the graph $\mathcal{S}_{2 n}(2)$; see [18]. Note that the case $k=2$ of Theorem 1 can be regarded as a special case of the classification in Theorem 3. The following special case of Theorem 3 has already been established in [4] by Francis Buekenhout and Xavier Hubaut.

Theorem 4 ([4], Theorem 2 (3)) Let $\Gamma$ be connected and locally $\mathcal{S} p_{2 n}(2)$ for some $n \geqslant 2$. Then $\Gamma$ is isomorphic to one of the following graphs $\mathcal{N}_{2 n+2}^{+}(2), \mathcal{N}_{2 n+2}^{-}(2)$, or $\mathcal{S} p_{2 n+2}(2)$ minus $\{x\} \cup x^{\perp}$ for some $x$.

The preceding theorem has been generalized in $[8,9]$.

\section{Local recognition of Weyl graphs}

We assume that the reader is familiar with Coxeter groups and root systems as treated in [20] or [5]. The commuting graph of a group $G$ on $X \subseteq G$ is the graph with vertex set $X$ in which two vertices $g, h \in X$ are adjacent whenever $g$ and $h$ commute. We will study the commuting graphs of finite Coxeter groups on their reflections. Since we are interested in local recognition results we will focus on finite irreducible Coxeter groups for which the reflection graph is locally homogeneous. The graphs arising from the cases $\mathrm{H}_{3}, \mathrm{H}_{4}$ and $\mathrm{I}_{2}(\mathrm{~m})$ are locally disjoint unions of complete graphs and therefore not interesting for the purpose of local recognition. Hence, we further restrict to Coxeter groups which arise from irreducible crystallographic root 
systems. These are those with Dynkin diagram equal to one of $A_{n}(n \geqslant 1), B_{n}$ or $C_{n}$ $(n \geqslant 2), D_{n}(n \geqslant 4), E_{6}, E_{7}, E_{8}, F_{4}$, or $G_{2}$.

Recall that each root of an irreducible crystallographic root system $\Phi$ is considered either short or long (with the convention that in the absence of two distinct root lengths every root is long). If $M$ is the Dynkin diagram of $\Phi$ then we denote with $W(M)$ the Weyl group of $\Phi$, i.e., the group generated by the reflections through the roots of $\Phi$, together with the notion of a short (respectively long) root reflection by $W(M)$. The Weyl graph $\mathbb{W}(M)$ is the commuting graph of $W(M)$ on its reflections. If $M$ is simply laced then all reflections in $W(M)$ are conjugate, which implies that the Weyl graph $\mathbb{W}(M)$ is locally homogeneous. On the other hand, if $M$ is not simply laced then there are two conjugacy classes of reflections in $W(M)$, namely short and long root reflections, and we regard $\mathbb{W}(M)$ as a bichromatic graph. Instead of assigning arbitrary colors we accordingly refer to the vertices of $\mathbb{W}(M)$ corresponding to short (respectively long) root reflections as short (respectively long) vertices. As a bichromatic graph, the Weyl graph $\mathbb{W}(M)$ is locally homogeneous.

$\mathbb{W}\left(A_{n}\right)$ is the graph with vertices $y_{i, j}, 1 \leqslant i<j \leqslant n+1$, such that $y_{i, j} \perp y_{k, l}$ if and only if $\{i, j\} \cap\{k, l\}=\emptyset$. Consequently, the Weyl graph $\mathbb{W}\left(A_{n}\right)$ is isomorphic to the Kneser graph $K(n+1,2)$. Likewise, $\mathbb{W}\left(D_{n}\right)$ is the graph with vertices $y_{i, j}$, $1 \leqslant i \neq j \leqslant n$, such that $y_{i, j} \perp y_{k, l}$ if and only if $\{i, j\} \cap\{k, l\}=\emptyset$ or $(k, l)=(j, i)$. $\mathbb{W}\left(D_{n}\right)$ is therefore isomorphic to the composition graph $K(n, 2)\left[K_{2}\right]$, that is, the graph arising from the Kneser graph $K(n, 2)$ by replacing each vertex by an adjacent pair of vertices. Accordingly, Theorem 1 applies and yields the recognition results of the Main Theorem for types $A_{n}$ and $D_{n}$. By [4] we have $\mathbb{W}\left(E_{6}\right) \cong \mathcal{N}_{6}^{-}(2), \mathbb{W}\left(E_{7}\right) \cong$ $\mathcal{S} p_{6}(2)$ and $\mathbb{W}\left(E_{8}\right) \cong \mathcal{N}_{8}^{+}(2)$. The corresponding recognition results of the Main Theorem follow from Theorems 1,3 and 4.

$\mathbb{W}\left(B_{n}\right)$ is the bichromatic graph with vertices $y_{i, j}, 1 \leqslant i, j \leqslant n$, where the $y_{i, i}$ are short and the $y_{i, j}$ with $i \neq j$ are long vertices, and $y_{i, j} \perp y_{k, l}$ if and only if $\{i, j\} \cap\{k, l\}=\emptyset$ or $(k, l)=(j, i)$. The Weyl graph $\mathbb{W}\left(C_{n}\right)$ is obtained from $\mathbb{W}\left(B_{n}\right)$ by exchanging the role of short and long vertices. The recognition results of the Main Theorem for types $B_{n}$ and $C_{n}$ are therefore contained in the following theorem.

Theorem 5 Let $n=3$ or $n \geqslant 5$, and let $\Gamma$ be a connected bichromatic graph which is locally like $\mathbb{W}\left(B_{n}\right)$. Then $\Gamma \cong \mathbb{W}\left(B_{n}\right)$.

Proof It is straightforward to check the case $n=3$.

Next, let $n \geqslant 6$. Let $X$ be a short component of $\Gamma$ and $x \in X$ a short vertex. The short induced subgraph on $x^{\perp}$ is a clique on $n-1$ elements which implies that $X$ is a clique on $n$ elements. By assumption, the long neighbors of $x$ induce a subgraph isomorphic to the long induced subgraph of $\mathbb{W}\left(B_{n-1}\right)$. This subgraph is isomorphic to $\mathbb{W}\left(D_{n-1}\right)$ and, in particular, is connected for $n \geqslant 6$. This implies that all long neighbors of $x$ are contained in a single long component $Y$ of $\Gamma$. Consider a short vertex $x_{1} \in X$ adjacent to $x$. Again, all long neighbors of $x_{1}$ lie in one long component of $\Gamma$. But looking at $\left\{x, x_{1}\right\}^{\perp} \subset x^{\perp}$ we see that $x$ and $x_{1}$ share long neighbors whence this component has to be $Y$ as well. Since $X$ is connected this shows that all long vertices adjacent to some vertex of $X$ are contained in $Y$. Likewise, let $y \in Y$. The short induced subgraph of $y^{\perp}$ is a clique on $n$ vertices and thus in particular connected. 
Again, we see that for a long vertex $y_{1}$ adjacent to $y$ the common neighbors $\left\{y, y_{1}\right\}^{\perp}$ contain a short vertex. Therefore the same argument as before shows that all short vertices adjacent to some vertex of $Y$ are contained in $X$. Since $\Gamma$ is connected this proves that $X$ and $Y$ are the only short respectively long components of $\Gamma$.

We count the number of long vertices by counting the long neighbors of the $n$ short vertices of $\Gamma$. By assumption, a short vertex has $(n-1)(n-2)$ long neighbors. Further, two short vertices have $(n-2)(n-3)$ long neighbors in common, three short vertices have $(n-3)(n-4)$ long neighbors in common, and so on. Thus there are

$$
\left(\begin{array}{l}
n \\
1
\end{array}\right)(n-1)(n-2)-\left(\begin{array}{l}
n \\
2
\end{array}\right)(n-2)(n-3)+\ldots+(-1)^{n-1}\left(\begin{array}{c}
n \\
n-2
\end{array}\right) 2=n(n-1)
$$

long vertices in $\Gamma$. Note that for the above equation we exploited that the alternating sum of the binomial coefficients equals zero, that is, $\sum_{k=0}^{n}(-1)^{k}\left(\begin{array}{l}n \\ k\end{array}\right)=0$.

Let $x_{1}, x_{2}, \ldots, x_{n}$ be the short vertices of $\Gamma . \Gamma$ is locally $\mathbb{W}\left(B_{n-1}\right)$ at short vertices which implies that for $1 \leqslant i \neq j \leqslant n$ the common neighborhood $\left\{x_{r}: r \notin\{i, j\}\right\}^{\perp}$ contains exactly two long vertices which we denote by $y_{i, j}$ and $y_{j, i}$. Since a long vertex is adjacent to exactly $n-2$ short vertices the $y_{i, j}$ thus defined are all distinct. By construction, $y_{i, j} \perp y_{j, i}$. Further, the $y_{i, j}$ exhaust $Y$ because $\Gamma$ contains exactly $n(n-$ 1) long vertices. Given two vertices $y_{i, j}$ and $y_{k, l}$, we find $m \in\{1,2, \ldots, n\} \backslash\{i, j, k, l\}$ whence $y_{i, j}$ and $y_{k, l}$ are both contained in $x_{m}^{\perp} \cong \mathbb{W}\left(B_{n-1}\right) . y_{i, j}$ is characterized in $x_{m}^{\perp}$ as one of the two long vertices contained in $\left\{x_{r}: r \notin\{i, j, m\}\right\}^{\perp}$. Likewise, $y_{k, l}$ is characterized in $x_{m}^{\perp}$ as one of the two long vertices contained in $\left\{x_{r}: r \notin\{k, l, m\}\right\}^{\perp}$. Consequently, for $\{i, j\} \neq\{k, l\}, y_{i, j} \perp y_{k, l}$ if and only if $\{i, j\} \cap\{k, l\}=\emptyset$. Hence, $\Gamma \cong \mathbb{W}\left(B_{n}\right)$.

Finally, consider the case $n=5$. We still find that each short component is a clique on 5 vertices. Let $X$ be one such short component. We count that there are 20 long vertices neighbored to one of the vertices of $X$. On the other hand, we see again that each long component has short neighbors in only one short component. Accordingly, the 20 long neighbors of $X$ constitute a union of long components. However, a long component is locally $K_{1} \sqcup 3 \cdot K_{2}$ and therefore has at least 12 vertices. We conclude that there is only one long component $Y$ with vertices neighbored to $X$. Now, the remainder of the preceding argument applies and shows that $\Gamma \cong \mathbb{W}\left(B_{5}\right)$ as claimed.

The case $n=4$ of Theorem 5 is discussed in Remark 12 where it is shown that there are infinitely many finite connected bichromatic graphs that are locally like $\mathbb{W}\left(B_{4}\right)$. The case of type $F_{4}$ is discussed in detail in the next section. Note that the Weyl graph $\mathbb{W}\left(G_{2}\right)$ is isomorphic to three disjoint edges of mixed type.

\section{Local recognition of $\mathbb{W}\left(F_{4}\right)$}

\subsection{Graphs locally like $\mathbb{W}\left(F_{4}\right)$}

The Weyl graph $\mathbb{W}\left(F_{4}\right)$ is a connected bichromatic locally homogeneous graph on 24 vertices with short local graph $\mathbb{W}\left(B_{3}\right)$ and long local graph $\mathbb{W}\left(C_{3}\right)$. As we will 
see shortly, $\mathbb{W}\left(F_{4}\right)$ is not locally recognizable. Before we turn to investigating additional constraints under which we seek to recognize $\mathbb{W}\left(F_{4}\right)$ nonetheless, we study connected bichromatic graphs $\Gamma$ which are locally like $\mathbb{W}\left(F_{4}\right)$. The results we obtain then guide our way in determining appropriate conditions under which we are able to recognize $\mathbb{W}\left(F_{4}\right)$ alongside its twisted copy. An easy but crucial observation to start with is the following.

Proposition 6 Let $\Gamma$ be locally like $\mathbb{W}\left(F_{4}\right)$. The short (respectively long) induced subgraph of $\Gamma$ is isomorphic to a disjoint union of 4-cliques.

Let $\Gamma$ be a bichromatic graph that is locally like $\mathbb{W}\left(F_{4}\right)$. Observe that the graph obtained from $\Gamma$ by exchanging the roles of short and long vertices is locally like $\mathbb{W}\left(F_{4}\right)$ as well. Results that we obtain for short vertices of graphs locally like $\mathbb{W}\left(F_{4}\right)$ are therefore also true for long vertices.

Paraphrasing Proposition 6, the vertices of $\Gamma$ come in 4-cliques of the same type. In order to simplify things it is natural to collapse these 4-cliques into single vertices.

Definition 7 Let $\Lambda$ be a graph and $\Pi$ a partition of its vertices. The contraction $\Lambda / \Pi$ is the graph on $\Pi$ such that two sets $A, B \in \Pi$ are adjacent whenever there is $a \in A$ and $b \in B$ which are adjacent in $\Lambda$. If $\Lambda$ is bichromatic then $\Pi$ is required to partition into sets of short and long vertices and $\Lambda / \Pi$ is a bichromatic graph in the natural way.

In this language, we thus investigate the collapsed graph $\Gamma / \Pi$ where $\Pi$ is the partition of $\Gamma$ into short and long 4-cliques. To this end, we analyze how these 4cliques relate to each other.

Proposition 8 Let $\Gamma$ be locally like $\mathbb{W}\left(F_{4}\right)$, and $x_{1}, x_{2}, x_{3}, x_{4}$ a short 4 -clique in $\Gamma$. Let $i \neq j$ and $k \neq l$.

- $\left\{x_{i}, x_{j}\right\}^{\perp}$ is locally $K_{2}^{s} \sqcup K_{2}^{\ell}$. In particular, for any pair $x_{i}, x_{j}$ there exist unique long vertices $y_{i, j}, y_{j, i}$ contained in $\left\{x_{i}, x_{j}\right\}^{\perp}$.

- $\left\{x_{i}, x_{j}, x_{k}\right\}^{\perp}$ contains no long vertex if $i, j, k$ are distinct. In particular, the vertices $y_{i, j}$ are all distinct.

- There are exactly 12 long vertices adjacent to at least one of the $x_{i}$, namely the above vertices $y_{i, j}$.

- $y_{i, j} \perp y_{k, l}$ implies that $\{k, l\}=\{i, j\}$ or $\{k, l\} \cap\{i, j\}=\emptyset$.

Proof Exploiting the local structure at $x_{i}$ we see that every short adjacent pair $x_{i}, x_{j}$ has exactly two long neighbors in common which we will (arbitrarily) denote by $y_{i, j}$ and $y_{j, i}$. Accordingly, $y_{i, j} \perp y_{j, i}$. Looking at the neighbors of a vertex $y_{i, j}$ reveals that $x_{i}$ and $x_{j}$ are the only short vertices among $x_{1}, x_{2}, x_{3}, x_{4}$ which are adjacent to $y_{i, j}$. Consequently, the $y_{i, j}$ are 12 distinct vertices. Since three adjacent short vertices share no long neighbors we count that exactly

$$
\left(\begin{array}{l}
4 \\
1
\end{array}\right) 6-\left(\begin{array}{l}
4 \\
2
\end{array}\right) 2=12
$$


long vertices are neighbored to at least one of the vertices $x_{1}, x_{2}, x_{3}, x_{4}$. Consequently, the long neighbors of the $x_{i}$ are precisely the vertices $y_{i, j}$. For the last claim, assume that $y_{i, j} \perp y_{k, l}$ and $\{k, l\} \cap\{i, j\}=\left\{i_{0}\right\}$. A look at the neighbors of $x_{i_{0}}$ shows that this is a contradiction.

If $\Gamma$ is locally like $\mathbb{W}\left(F_{4}\right)$ and $\Pi$ is the partition of $\Gamma$ into short and long 4-cliques, then we add the following extra structure to the collapsed graph $\Gamma / \Pi$. Two vertices $X, Y \in \Gamma / \Pi$ are said to be strongly connected if every $x \in X$ is at distance 1 from $Y$ in $\Gamma$ and vice versa. In this case, we think of $X$ and $Y$ as being connected by two edges, the reason of which will be clear from the next proposition. The number of neighbors of $X$ where we count those neighbors twice that are strongly connected to $X$ is said to be the bivalency of $X$.

Proposition 9 Let $\Gamma$ be locally like $\mathbb{W}\left(F_{4}\right)$, and let $\Pi$ be the partition of $\Gamma$ into short and long 4-cliques. The contraction $\Gamma / \Pi$ is bipartite of bivalency 6 .

Proof Let $X \in \Gamma / \Pi$ be a short vertex. By Proposition 6, $X$ has only long neighbors. $X=\left\{x_{1}, x_{2}, x_{3}, x_{4}\right\}$ is a 4-clique of $\Gamma$ and according to Proposition 8 there are 12 long vertices $y_{i, j}$ at distance 1 from $X$ in $\Gamma$. Each pair of vertices $y_{i, j}, y_{j, i}$ is contained in exactly one long neighbor $Y_{\{i, j\}}$ of $X$. Let $k, l$ be the indices such that $\{i, j, k, l\}=$ $\{1,2,3,4\}$. Then, by Proposition 8, either $Y_{\{k, l\}} \neq Y_{\{i, j\}}$, in which case both long vertices are connected to $X$ by just one edge, or $Y_{\{k, l\}}=Y_{\{i, j\}}$, in which case both long vertices are connected to $X$ by two edges. In any case, we count that the bivalency of $X$ is 6 .

We now do the reverse and prove that every bipartite graph of bivalency 6 is the contraction of some graph which is locally like $\mathbb{W}\left(F_{4}\right)$. Note, however, that nonisomorphic graphs locally like $\mathbb{W}\left(F_{4}\right)$ can have isomorphic contractions.

Lemma 10 For every connected bipartite graph $\Lambda$ of bivalency 6 there is a connected bichromatic graph $\Gamma$ that is locally like $\mathbb{W}\left(F_{4}\right)$ such that $\Gamma / \Pi=\Lambda$ where $\Pi$ is the partition of $\Gamma$ into short and long 4-cliques.

Proof Let $\Lambda$ be a bipartite graph of bivalency 6. Exploiting that $\Lambda$ is 2-colorable, we may identify $\Lambda$ with a bichromatic graph such that no two vertices of the same type are adjacent. For any vertex $x$ of $\Lambda$ choose an injection

$$
x^{\perp} \rightarrow\left(\begin{array}{l}
4 \\
2
\end{array}\right), \quad y \mapsto a(x, y)
$$

from its neighbors to the six 2-subsets of $\{1,2,3,4\}$ such that for strongly connected vertices $x, y$ the complement of $a(x, y)$ is not attained. This is always possible since $\Lambda$ has bivalency 6 . To every directed edge $(x, y)$ we thus assigned the 2 -subset $a(x, y)$ of $\{1,2,3,4\}$. Construct the bichromatic graph $\Gamma$ from $\Lambda$ as follows. For every vertex $x \in \Lambda$ add a 4-clique $x_{1}, x_{2}, x_{3}, x_{4}$ of the same type as $x$ to $\Gamma$. Then, for $x, y \in \Lambda$ let $x_{i}$ and $y_{j}$ be adjacent in $\Gamma$ if and only if $x$ and $y$ are adjacent in $\Lambda$ and the following holds: either $(i, j) \in a(x, y) \times a(y, x)$, or $x$ and $y$ are strongly 
connected and $(i, j) \in \overline{a(x, y)} \times \overline{a(y, x)}$. By construction, contracting the 4-cliques of $\Gamma$ produces $\Lambda$. It is straightforward to check that $\Gamma$ is locally like $\mathbb{W}\left(F_{4}\right)$.

Corollary 11 There exist infinitely many non-isomorphic finite connected bichromatic graphs that are locally like $\mathbb{W}\left(F_{4}\right)$.

Proof We claim that there are infinitely many finite connected bipartite graphs $\Lambda$ that are locally $\overline{K_{6}}$ and hence of bivalency 6 (if no vertices are assumed to be strongly connected). To this end, note that the graphs $C_{k} \times C_{m} \times C_{n}$ are connected and locally $\overline{K_{6}}$ for $k, m, n \geqslant 4$. Since cycles $C_{n}$ are 2-colorable whenever $n$ is even, the graphs $C_{k} \times C_{m} \times C_{n}$ are 2-colorable and hence bipartite whenever $k, m, n$ are all even. The claim follows from Lemma 10.

Remark 12 Analogous to Lemma 10 one shows that for every connected bipartite graph $\Lambda$ of bivalency $(2,6)$ (meaning that vertices of one type have valency 2 and vertices of the other type have valency 6) there is a connected bichromatic graph $\Gamma$ that is locally like $\mathbb{W}\left(B_{4}\right)$ such that $\Gamma / \Pi=\Lambda$ where $\Pi$ is again the partition of $\Gamma$ into short and long 4-cliques. This easily implies that there are infinitely many finite connected bichromatic graphs that are locally like $\mathbb{W}\left(B_{4}\right)$.

Let $\Gamma$ be locally like $\mathbb{W}\left(F_{4}\right)$ and assume that the collapsed graph $\Gamma / \Pi$ contains strongly connected vertices $X$ and $Y$. This means that, say, $X=x_{1}, x_{2}, x_{3}, x_{4}$ are short vertices, $Y=y_{1}, y_{2}, y_{3}, y_{4}$ are long vertices, and we have the adjacencies

$$
x_{1}, x_{2} \perp y_{1}, y_{2}, \quad x_{3}, x_{4} \perp y_{3}, y_{4} .
$$

It is straightforward to check that replacing these by

$$
x_{1}, x_{2} \perp y_{3}, y_{4}, \quad x_{3}, x_{4} \perp y_{1}, y_{2}
$$

produces a graph $\Gamma^{\prime}$ which is also locally like $\mathbb{W}\left(F_{4}\right)$. We say that $\Gamma^{\prime}$ is a twisted copy of $\Gamma$. In particular, for $\Gamma=\mathbb{W}\left(F_{4}\right)$ these twisted copies are all isomorphic and we denote the resulting graph by $\mathbb{W}\left(F_{4}\right)^{\prime}$.

\subsection{Recognition results}

We now discuss further properties of the Weyl graph $\mathbb{W}\left(F_{4}\right)$ in order to characterize $\mathbb{W}\left(F_{4}\right)$ among the connected bichromatic graphs that are locally like $\mathbb{W}\left(F_{4}\right)$. For more details we refer to the thesis [28] of the third author. We start with some easy observations.

Proposition 13 Let $\Gamma$ be a finite bichromatic graph that is locally like $\mathbb{W}\left(F_{4}\right)$. Then the numbers of short and long vertices in $\Gamma$ are the same, $|\Gamma|$ is divisible by 8 and $|\Gamma| \geqslant 24$.

Since $\left|\mathbb{W}\left(F_{4}\right)\right|=24$ we see that, in a sense, $\mathbb{W}\left(F_{4}\right)$ is maximally tight among the graphs that are locally like $\mathbb{W}\left(F_{4}\right)$. There are several further properties of a graph, for instance its diameter, that describe its tightness. The following notion of tight connectedness is another way to express tightness of a bichromatic graph. 
Definition 14 A bichromatic graph is said to be tightly connected if every long vertex has a neighbor in every short component and vice versa.

These three notions of tightness, however, are not local in nature (where a local property is meant to be one which can be expressed in terms of the neighbors of each vertex). In order to find a more local notion to describe the tightness of $\mathbb{W}\left(F_{4}\right)$ we investigate the relation of vertices at distance 2 . The following is straightforward to check.

Proposition 15 Let $\Gamma$ be locally like $\mathbb{W}\left(F_{4}\right)$, and let $x, y \in \Gamma$ be at distance 2.

- If $x, y$ are both short (respectively long) vertices then $\{x, y\}^{\perp} \cong \mu(x, y) \cdot K_{1}^{\ell}$ (respectively $\left.K_{1}^{s}\right)$ for some $\mu(x, y) \in\{1,2,3\}$.

- If $x, y$ are of mixed type then $\{x, y\}^{\perp} \cong \mu_{s}(x, y) \cdot K_{2}^{s} \sqcup \mu_{\ell}(x, y) \cdot K_{2}^{\ell}$ for some $\mu_{s}(x, y)+\mu_{\ell}(x, y) \in\{1,2\}$.

For the Weyl graph $\mathbb{W}\left(F_{4}\right)$ the parameters $\mu, \mu_{s}, \mu_{\ell}$ defined in Proposition 15 are constant and take the maximum possible values $\mu=3$ and $\mu_{s}=\mu_{\ell}=1$ which is another, more local, instantiation of the tightness of $\mathbb{W}\left(F_{4}\right)$. Notice that the condition $\mu_{s}=\mu_{\ell}=1$ is equivalent to the contraction $\Gamma / \Pi$, studied in Proposition 9 , being locally homogeneous with $\Delta_{s}(\Gamma / \Pi) \cong{\overline{K_{3}}}^{\ell}$ and $\Delta_{\ell}(\Gamma / \Pi) \cong{\overline{K_{3}}}^{s}$.

The following theorem summarizes our recognition results for the Weyl graph $\mathbb{W}\left(F_{4}\right)$. Note that all of the provided conditions under which a graph $\Gamma$ is almost recognized as $\mathbb{W}\left(F_{4}\right)$ are statements which describe the tightness of $\Gamma$.

Theorem 16 Let $\Gamma$ be a connected bichromatic graph that is locally like $\mathbb{W}\left(F_{4}\right)$. Assume that

- $|\Gamma|=24$, or

- $\Gamma$ is tightly connected, or

- $\Gamma$ has diameter 2 , or

- $\mu=3$.

If one of these conditions holds then $\Gamma$ is isomorphic to $\mathbb{W}\left(F_{4}\right)$ or to its twisted copy $\mathbb{W}\left(F_{4}\right)^{\prime}$. In particular, Aut $(\Gamma) \cong W\left(F_{4}\right) / Z$ where $Z$ denotes the center of $W\left(F_{4}\right)$.

We prove Theorem 16 by a series of propositions. The proof of the case $\mu=3$ is similar in spirit to the previous ones. It is therefore omitted; the interested reader is referred to [28] for the details.

Proposition 17 Let $\Gamma$ be a connected bichromatic graph that is locally like $\mathbb{W}\left(F_{4}\right)$. If $|\Gamma|=24$ then $\Gamma \cong \mathbb{W}\left(F_{4}\right)$ or $\Gamma \cong \mathbb{W}\left(F_{4}\right)^{\prime}$. Further, Aut $(\Gamma) \cong W\left(F_{4}\right) / Z$.

Proof As observed in Proposition 13, every graph that is locally like $\mathbb{W}\left(F_{4}\right)$ has at least 12 short and 12 long vertices. $\Gamma$ therefore consists of exactly 12 vertices of each type.

Let $x_{1}, x_{2}, x_{3}, x_{4}$ be a short 4-clique. Adopting the notation of Proposition 8 , let $y_{i, j}$ and $y_{j, i}$ be the long vertices adjacent to both $x_{i}$ and $x_{j}$. The $y_{i, j}$ are 12 distinct 
vertices and therefore constitute the long vertices of $\Gamma$. It follows from Proposition 8 that the three long 4-cliques are given by $y_{i, j}, y_{j, i}, y_{k, l}, y_{l, k}$ for disjoint $\{i, j\}$ and $\{k, l\}$.

Each of the remaining eight short vertices has exactly two long neighbors in each of the three long 4-cliques. Let $x_{5}$ be one of remaining short vertices. The two neighbors of $x_{5}$ in a 4-clique $y_{i, j}, y_{j, i}, y_{k, l}, y_{l, k}$ are one of $y_{i, j}, y_{j, i}$ along with one of $y_{k, l}, y_{l, k}$. We ambiguously defined the vertices $y_{i, j}, y_{j, i}$ as the long vertices contained in $\left\{x_{i}, x_{j}\right\}^{\perp}$ so we may as well assume that $x_{5}$ is adjacent to $y_{i, j}$ and $y_{k, l}$ with $i<j$ and $k<l$. Let $x_{6}$ be the unique short vertex also adjacent to $y_{1,2}, y_{3,4}$. Likewise, let $x_{7}$ be the short vertex also adjacent to $y_{1,3}, y_{2,4}$, and $x_{8}$ the short vertex also adjacent to $y_{1,4}, y_{2,3}$. By construction, $x_{5}, x_{6}, x_{7}, x_{8}$ is a 4-clique. Notice that for instance $x_{5}, x_{6} \in\left\{y_{1,2}, y_{3,4}\right\}^{\perp}$ implies that $x_{7}, x_{8} \in\left\{y_{2,1}, y_{4,3}\right\}^{\perp}$. Altogether this determines the induced subgraph on $x_{1}, x_{2}, \ldots, x_{8}$ along with the vertices $y_{i, j}$.

Let $x_{9}, x_{10}, x_{11}, x_{12}$ be the remaining short 4-clique. We may assume that $x_{9}, x_{10}$ are the short vertices contained in $\left\{y_{1,2}, y_{4,3}\right\}^{\perp}$. Accordingly, $x_{11}, x_{12} \in\left\{y_{2,1}, y_{3,4}\right\}^{\perp}$. We may also assume that $x_{9}$ is contained in $\left\{y_{1,3}, y_{4,2}\right\}^{\perp}$ (because if both $x_{9}$ and $x_{10}$ were not contained in $\left\{y_{1,3}, y_{4,2}\right\}^{\perp}$ then both $x_{11}, x_{12} \in\left\{y_{1,3}, y_{4,2}\right\}^{\perp}$ which contradicts $\left.x_{11}, x_{12} \in\left\{y_{2,1}, y_{3,4}\right\}^{\perp}\right)$. Further, we may assume that $x_{11}$ is the second short vertex contained in $\left\{y_{1,3}, y_{4,2}\right\}^{\perp}$. Consider the two short vertices in $\left\{y_{1,4}, y_{3,2}\right\}^{\perp}$. These can be either $x_{9}, x_{12}$ or $x_{10}, x_{11}$, and either choice determines $\Gamma$. Denote with $\Gamma_{1}$ the graph corresponding to the choice $x_{9}, x_{12} \in\left\{y_{1,4}, y_{3,2}\right\}^{\perp}$, and with $\Gamma_{2}$ the graph corresponding to the choice $x_{10}, x_{11} \in\left\{y_{1,4}, y_{3,2}\right\}^{\perp}$. The following table summarizes adjacency involving the vertices $x_{9}, x_{10}, x_{11}, x_{12}$.

$\begin{array}{lll}\text { by construction } & x_{9}, x_{10} \perp y_{1,2}, y_{4,3} & x_{11}, x_{12} \perp y_{2,1}, y_{3,4} \\ & x_{9}, x_{11} \perp y_{1,3}, y_{4,2} & x_{10}, x_{12} \perp y_{3,1}, y_{2,4} \\ \Gamma_{1} & x_{9}, x_{12} \perp y_{1,4}, y_{3,2} & x_{10}, x_{11} \perp y_{4,1}, y_{2,3} \\ \Gamma_{2} & x_{9}, x_{12} \perp y_{4,1}, y_{2,3} & x_{10}, x_{11} \perp y_{1,4}, y_{3,2}\end{array}$

An implementation in the computer algebra system SAGE, see [26], of the graphs $\Gamma_{1}$ and $\Gamma_{2}$ can be found in the appendix of [28]. In particular, it is verified that $\Gamma_{1}$ and $\Gamma_{2}$ are non-isomorphic, that the automorphism group of both graphs is isomorphic to $W\left(F_{4}\right) / Z$, and that $\Gamma_{1}$ is isomorphic to $\mathbb{W}\left(F_{4}\right)$. Accordingly, $\Gamma_{2}$ is isomorphic to the twisted copy $\mathbb{W}\left(F_{4}\right)^{\prime}$.

Proposition 18 Let $\Gamma$ be a connected bichromatic graph that is locally like $\mathbb{W}\left(F_{4}\right)$. If $\Gamma$ is tightly connected then $\Gamma \cong \mathbb{W}\left(F_{4}\right)$ or $\Gamma \cong \mathbb{W}\left(F_{4}\right)^{\prime}$.

Proof Fix a short 4-clique $x_{1}, x_{2}, x_{3}, x_{4}$. Because of tightness every long vertex is adjacent to one of the $x_{i}$, and by Proposition 8 there are exactly 12 such long vertices. Thus $\Gamma$ consists of 12 long vertices. Likewise, $\Gamma$ contains exactly 12 short vertices. Hence, $|\Gamma|=24$, and the claim follows from Proposition 17.

Proposition 19 Let $\Gamma$ be a connected bichromatic graph that is locally like $\mathbb{W}\left(F_{4}\right)$. If $\Gamma$ has diameter 2 then $\Gamma \cong \mathbb{W}\left(F_{4}\right)$ or $\Gamma \cong \mathbb{W}\left(F_{4}\right)^{\prime}$.

Proof Let $x_{1}, x_{2}, x_{3}, x_{4}$ be a short 4-clique. As in Proposition 8 let $y_{i, j}, y_{j, i}$ be the long vertices adjacent to both $x_{i}$ and $x_{j}$. Assume that there is a long vertex $v$ which 
is not among the 12 long vertices $y_{i, j}$. Because $v$ is not adjacent to any of the $x_{i}$ and since the diameter of $\Gamma$ is 2 , we find a long vertex that connects $x_{1}$ and $v$. Without loss of generality let this long vertex be $y_{1,2}$. This prevents $y_{1,2}, y_{2,1}, y_{3,4}, y_{4,3}$ from forming a long 4-clique. By Proposition 8 there are thus long vertices $v_{1}, v_{2}$ not among the $y_{i, j}$ such that $y_{3,4}, y_{4,3}, v_{1}, v_{2}$ form a long 4-clique. Again, $v_{1}$ is not adjacent to any of the $x_{i}$ and hence is connected to $x_{1}$ by a long vertex. This is a contradiction since the long vertices adjacent to $x_{1}$ are the vertices $y_{1, j}, y_{j, 1}$.

Consequently, $\Gamma$ contains no further long vertices besides the 12 vertices $y_{i, j}$, and hence, by Proposition 13, $|\Gamma|=24$. Apply Proposition 17.

\section{Group-theoretic applications}

Our guiding example for the application of the local recognition of graphs in group theory is the characterization of the symmetric groups by means of the structure of its transposition centralizers; cf. [10, Theorem 27.1]. A detailed proof of [10, Theorem 27.1] using local recognition results for the Weyl graphs of type $A_{n}$ is contained in the third author's thesis [28]; that proof runs along the lines of the proof of [7, Theorem 1.2]. An early example of such results can be found in [21] which along with [4] has been one of the original motivations for the second author to pursue the local recognition of Kneser graphs in [16]. Another fundamental example for this theme is [22].

Likewise, the recognition results for the Weyl graph of type $F_{4}$ discussed in the previous section give rise to the following local characterization of the Weyl group $W\left(F_{4}\right)$. Again, we refer to [28] for a proof inspired by [7].

Theorem 20 Let $G$ be a group with non-conjugate involutions $x, y$ such that

- $C_{G}(x)=\langle x\rangle \times J$ with $J \cong W\left(B_{3}\right)$,

- $C_{G}(y)=\langle y\rangle \times K$ with $K \cong W\left(C_{3}\right)$,

- $x$ (respectively $y$ ) is a short (respectively long) root reflection in $K$ (respectively $J)$,

- $J \cap K$ contains involutions $x_{1}, y_{1}$ such that $x_{1}$ (respectively $y_{1}$ ) is a short (respectively long) root reflection in $K$ as well as in $J$, and

- there are a long root reflection $y_{0} \neq y, y_{1}$ in $J$ and a short root reflection $x_{0} \neq x, x_{1}$ in $K$ such that $x_{0}$ and $y_{0}$ commute.

If $G=\langle J, K\rangle$ then $G \cong W\left(F_{4}\right)$.

The interest in group-theoretic local recognition results like Theorem 20 stems from the classification of finite simple groups (outlined in [10]) and the fact that the majority of finite simple groups arises from (twisted) Chevalley groups. These can be defined as groups generated by subgroups isomorphic to $\operatorname{SL}(2, q)$ subject to certain relations by the Curtis-Tits theorem formulated as in [19, 23, 29], by Phan's theorems [24, 25], and by the Phan-type theorems [2, 12, 13]. Recently, see [11, Local recognition theorem 1], Kristina Altmann and the first author proved a local recognition result for Chevalley groups of (twisted) type $A_{7}$ and $E_{6}$ based on results 
and techniques of [1]; this result makes serious use of the local recognition of graphs that are locally the Weyl graph of type $A_{5}$ and of the Curtis-Tits theorem and Phan's theorems. We hope that our analysis can help to approach a similar recognition result for Chevalley groups of type $F_{4}$ based on the Phan-type theorem of type $F_{4}$ proved by Hoffman, Mühlherr, Shpectorov and the first author and published in [13]. For more details we refer to the thesis [28] of the third author and the survey [11] of the first author.

Acknowledgements The authors thank the referee for several suggestions that helped to improve the exposition of this article.

\section{References}

1. Altmann, K.: Centralisers of fundamental subgroups. PhD thesis, Technische Universität Darmstadt (2007)

2. Bennett, C.D., Gramlich, R., Hoffman, C., Shpectorov, S.: Odd-dimensional orthogonal groups as amalgams of unitary groups, part 1: general simple connectedness. J. Algebra 312, 426-444 (2007)

3. Brown, M., Connelly, R.: On graphs with a constant link, II. Discrete Math. 11, 199-232 (1975)

4. Buekenhout, F., Hubaut, X.: Locally polar spaces and related rank 3 groups. J. Algebra 45, 391-434 (1977)

5. Bourbaki, N.: Elements of Mathematics. Lie Groups and Lie Algebras: Chapters 4-6. Springer, Berlin (2002)

6. Cohen, A.M.: Local recognition of graphs, buildings, and related geometries. In: Kantor W.M., Liebler R.A., Payne S.E., Shult E.E. (eds.) Finite Geometries, Buildings, and Related Topics, pp. 85-94. Oxford Science Publications, The Clarendon Press, New York (1990)

7. Cohen, A.M., Cuypers, H., Gramlich, R.: Local recognition of non-incident point-hyperplane graphs. Combinatorica 25, 271-296 (2005)

8. Cohen, A.M., Shult, E.E.: Affine polar spaces. Geom. Dedicata 35, 43-76 (1990)

9. Cuypers, H., Pasini, A.: Locally polar geometries with affine planes. European J. Combin. 13, 39-57 (1992)

10. Gorenstein, D., Lyons, R., Solomon, R.: The Classification of the Finite Simple Groups. AMS, Providence (1994)

11. Gramlich, R.: Developments in finite Phan theory. Innov. Incidence Geom. To appear

12. Gramlich, R., Hoffman, C., Shpectorov, S.: A Phan-type theorem for $\operatorname{Sp}(2 n, q)$. J. Algebra 264, 358384 (2003)

13. Gramlich, R., Witzel, S.: The sphericity of the Phan geometries of type $B_{n}$ and $C_{n}$ and the Phan-type theorem of type $F_{4}$. Submitted. arXiv:0901.1156

14. Hall, J.I.: Locally Petersen graphs. J. Graph Theory 4, 173-187 (1980)

15. Hall, J.I.: Graphs with constant link and small degree or order. J. Graph Theory 8, 419-444 (1985)

16. Hall, J.I.: A local characterization of the Johnson scheme. Combinatorica 7, 77-85 (1987)

17. Harary, F.: Graph Theory. Westview Press (1994)

18. Hall, J.I., Shult, E.E.: Locally cotriangular graphs. Geom. Dedicata 18, 113-159 (1985)

19. Humphreys, J.E.: Remarks on "A theorem on special linear groups". J. Algebra 22, 316-318 (1972)

20. Humphreys, J.E.: Reflection Groups and Coxeter Groups. Cambridge University Press, Cambridge (1992)

21. Mullineux, G.: A characterization of $A_{n}$ by centralizers of short involutions. Quart. J. Math. Oxford Ser. 29, 213-220 (1978)

22. Pasechnik, D.V.: Geometric characterization of the sporadic groups $\mathrm{Fi}_{22}, \mathrm{Fi}_{23}$, and $\mathrm{Fi}_{24}$. J. Combin. Theory Ser. A 68, 100-114 (1994)

23. Phan, K.-W.: A theorem on special linear groups. J. Algebra 16, 509-518 (1970)

24. Phan, K.-W.: On groups genererated by three-dimensional special unitary groups, I. J. Austral. Math. Soc. Ser. A 23, 67-77 (1977)

25. Phan, K.-W.: On groups genererated by three-dimensional special unitary groups, II. J. Austral. Math. Soc. Ser. A 23, 129-146 (1977) 
26. SAGE Mathematical Software: Version 2.8. http://www.sagemath.org (2007)

27. Shult, E.E.: Groups, polar spaces and related structures. In: Proc. Advanced Study Inst., Breukelen, 1975. Math. Centre Tracts, vol. 57, pp. 130-161. Math. Centrum, Amsterdam (1974)

28. Straub, A.: Local recognition of reflection graphs on Coxeter groups. Master's thesis, Technische Universität Darmstadt. arXiv:0805.2403 (2008)

29. Timmesfeld, F.G.: The Curtis-Tits presentation. Adv. Math. 189, 38-67 (2004)

30. Weetman, G.: A construction of locally homogeneous graphs. J. London Math. Soc. 50, 68-86 (1994)

31. Weetman, G.: Diameter bounds for graphs extensions. J. London Math. Soc. 50, 209-221 (1994) 